Communications in Physics, Vol. 24, No. 1 (2014), pp. 85-96

\title{
INFLUENCE OF BLOCKING EFFECT AND ENERGETIC DISORDER ON DIFFUSION IN ONE-DIMENSIONAL LATTICE
}

\author{
MAI THI LAN, NGUYEN VAN HONG, NGUYEN THU NHAN \\ Hanoi University of Science and Technology, No 1, Dai Co Viet, Hanoi, Vietnam \\ HOANG VAN HUE \\ Ho Chi Minh City University of Food Industry \\ E-mail: lan.maithi@hust.edu.vn
}

Received 28 November 2013

Accepted for publication 22 March 2014

\begin{abstract}
The diffusion in one-dimensional disordered lattice with Gaussian distribution of site and transition energies has been studied by mean of kinetic Monte-Carlo simulation. We focus on investigating the influence of energetic disorders and diffusive particle density on diffusivity. In single-particle case, we used both analytical method and kinetic Monte-Carlo simulation to calculate the quantities that relate to diffusive behavior in disordered systems such as the mean time between two consecutive jumps, correlation factor and diffusion coefficient. The calculation shows a good agreement between analytical and simulation results for all disordered lattice types. In many-particle case, the blocking effect results in decreasing correlation factor $F$ and average time $\tau_{\text {jump }}$ between two consecutive jumps. With increasing the number of particles, the diffusion coefficient $D_{M}$ decreases for site-energy and transition-energy disordered lattices due to the F-effect affects stronger than $\tau$-effect. Furthermore, the blocking effect almost is temperature independent for both lattices.
\end{abstract}

Keywords: diffusion, disordered lattices, Gaussian distribution, blocking effect, energetic disorders.

\section{INTRODUCTION}

The diffusion in disordered lattices exhibits a variety of interesting phenomena, such as a strong reduction of the asymptotic diffusion coefficient, anomalous frequency dependence of the conductivity, dispersive transport, etc [1]. It also received wide attention from researchers in the fields related to the fuel cells, membrane technology, nano devices...[2-11]. Recently, a significant amount of experimental data pertaining to real materials is available. Experimental data, together with simulation results, will allow us to get insight into the diffusivity in strongly disordered systems [12]. However, the number of theoretical works relating to diffusion in disordered lattices is very limited, particularly in higher-dimensional disordered lattices. Moreover, most of works uses very simple models (such as models of disordered lattices with two-level distribution of site and transition (saddle point) energies) [6-12].

Up to now, the exact determination of diffusion coefficient, as well as the diffusive behavior at short time interval for disordered materials is still unclear. Diffusion in materials was influenced (C)2014 Vietnam Academy of Science and Technology 
by both geometrical and energetic disorder but mainly by the energetic disorder. Hence, to clarify the diffusive behavior of disordered systems, the disordered lattice is employed where the particle jumps between sites of regular lattice whose site and barrier energy are randomly distributed.

In this paper we focus on studying the influence of energetic disorder on the diffusive behavior. The simulation has been conducted for a chain consisting of 4000 sites with periodic boundary conditions. Three lattice types are considered including: the first one is the site disordered lattice (SDL) where the transition energies are constant but site energies are adopted in accordance to Gaussian distribution [13]; the second one is transition disordered lattice (TDL) where the transition energies are adopted in accordance to Gaussian distribution and site energies are kept constant; and the third one is mixed disordered lattice (MDL) where both site and transition energies are adopted in accordance to Gaussian distribution.

\section{CALCUlation METHOD}

Let us consider the hopping of particles between sites in one-dimensional disordered lattice. Each $i^{\text {th }}$ site is characterized by its energy $E_{i}$. The hopping of particle from the $i^{t h}$ site to the nearest neighbor ones $i-1$ and $i+1$ is described by the transition energy $E_{i, i-1}$ and $E_{i, i+1}$, respectively. The transition and site energies are assigned random values obeying Gaussian distribution as follows:

$$
p(E)=\frac{1}{\sigma \sqrt{2 \pi}} \exp \left(\frac{-(E-\mu)^{2}}{2 \sigma^{2}}\right)
$$

In order to simply the problem, we use the standard Gaussian distribution with the parameter $\mu=0 ; \sigma=1$ and it can be expressed as follows:

$$
p(E)=\frac{1}{\sqrt{2 \pi}} \exp \left(-\frac{E^{2}}{2}\right) \quad, \text { with } \int_{-5}^{5} p(E) d E=1
$$

Here $E \equiv E_{x}$ (with the index $x$ is $s$ or $t$ ) is the site energy $E_{s}$ or transition energy $E_{t}$, respectively. Once the particle presents at site $i$, its probability to hop into neighboring site $i \pm 1$ is given by

$$
p_{i, i+1}=\frac{\exp \left(-E_{i, i \pm 1} \beta\right)}{\exp \left(-E_{i, i+1} \beta\right)+\exp \left(-E_{i, i-1} \beta\right)}
$$

The particle's jump from one site to another is a Poisson process with average delay time is

$$
\tau_{i}=\frac{2 \tau_{0} \exp \left(-E_{i} \beta\right)}{\exp \left(-E_{i, i+1} \beta\right)+\exp \left(-E_{i, i-1} \beta\right)}
$$

where $\tau_{0}$ is frequency (period); $\beta=1 / k_{B} T ; k_{B}$ is Boltzmann constant, and $T$ is temperature. In fact, the time $\tau_{i}$ is the mean residence time of particle on site $i$.

The Monte-Carlo (MC) method with the Metropolis algorithm and a lot of other algorithms built on it are at the heart of many, if not most, of the simulations investigating properties of physical systems at stationary states.

In this paper, we applied the Monte-Carlo simulation called "residence time" method or kinetic Monte-Carlo (KMC) method. The KMC method developed on base of a different kind of Monte Carlo algorithm for evolving systems dynamically from state to state and in principle, it can give the exact dynamical evolution of a system. More detail about KMC can be found elsewhere 
in $[14,15]$. In this method each MC step leads to hopping of particle, but random sampling determines the time that particle spent on site $i$ where it visits. After construction of the lattice, the sites are filled with $N_{p}$ particles by randomly choosing their coordinates and avoiding double occupancy. The algorithm consists of five main steps are:

1) determine the residence time of particle on the current site $i$ by

$$
\tau_{i j}=-\tau_{i} \ln R
$$

where $R$ is random number in range $[0,1]$.

Initially, a list of time $\tau_{\text {jumpj }}, j=1,2, \ldots N_{p}$ is determined by equation (5) $\tau_{j u m p j}=\tau_{i j}=-\tau_{i} \ln R$ :

2) select a particle $\mathrm{j}$ based on the list $\tau_{j u m p j}$. The particle performing next hop is one that has the earliest time from this list (the smallest value from the list of $\tau_{j u m p j}$ );

3 ) select the hop direction of the particle $j$ (to left or right site) according to probability $\mathrm{p}_{i, i \pm 1}$ (see Eq. (3));

3) move the particle $\mathrm{j}$ into the corresponding neighboring site if this site is non-occupied. Otherwise the particle remains at current site $i$;

5) the time $\tau_{\text {jumpj }}$ is added to

$$
\tau_{j u m p j}=\tau_{j u m p j}-\tau_{i} \ln R .
$$

The total duration of the trajectory is given by $t_{n}=\sum \tau_{i j}$. The mean time between two consecutive jumps is $\tau_{\text {jumpy }}=<t_{n}>/ n$. During simulation the mean square displacement $<x_{n}^{2}>$ is obtained by averaging over many runs. Correlation factor $F_{y}$ is the slopes of the linear dependence $<x_{n}^{2}>$ vs. $n$. Once given a time $<t_{n}>$ that is the average duration of $n$ MC steps, the diffusion coefficient can be calculated by

$$
D_{y}=\frac{a^{2}}{2} \frac{F_{y}}{\tau_{\text {jumpy }}} .
$$

Here $a$ is distance between the nearest neighboring sites. The letter $y$ may be $S, M$ or $C$ corresponding to single-particle, many-particle and crystal case, respectively. The crystal case corresponds to the lattice where site and transition energies are constant.

The simulation has conducted for two types of one-dimensional lattices consisting of 4000 sites with periodic boundary conditions. The values of parameters used for calculation are the same for all simulations and given as follow: the temperature, $\xi=\sigma \beta ; \xi$ is dimensionless and varies in the range from 0.2 to 1.2 . The average number of hops per particle is $n=1000$; the number of particles is varies from 1 to 120 particles. In order to obtain a good statistics, all quantities is obtained by averaging over $10^{6} \mathrm{MC}$ samplings.

\section{ANALYTIC EXPRESSION FOR THE DIFFUSION QUANTITIES IN THE CASE OF SINGLE-PARTICLE}

At first we introduce some necessary parameters for further discussion. The time for performing $n$ jump steps is denoted as $t_{n}$ and the mean time between two consecutive jumps is $\tau_{\text {jump }}=<t_{n}>/ n$. Here $<t_{n}>$ is the average time $t_{n}$ over many simulations.

In the case of SDL system: $\left\langle x_{n}^{2}\right\rangle=n a^{2}$. Meanwhile, in the case of TD system, due to forward and backward jumps $<x_{n}^{2}>=F n a^{2}$ with $F<1$. The correlation factor $\mathrm{F}$ which has very 
(a)

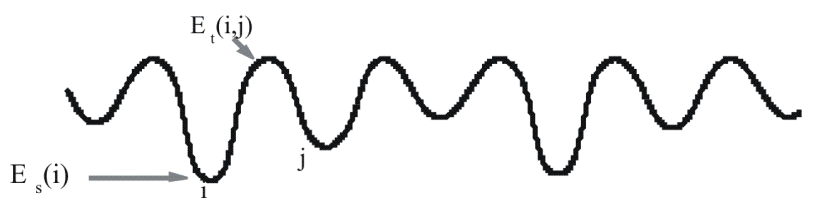

(b)

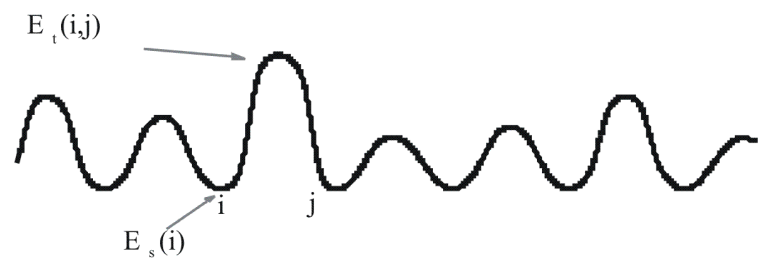

(c)

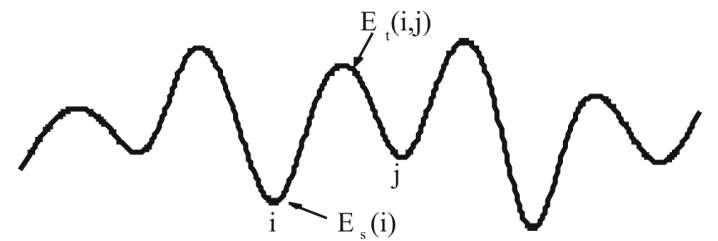

Fig. 1. Distribution of site and transition energy in (a) SDL, (b) TDL and (c) MDL

small value at low temperature. Consider the single-particle case when particle walks randomly for a long time $t_{n}$. In this case, the time that particle spends at each $i^{t h}$ site can be approximated by

$$
t_{i}=t_{n} \frac{\exp \left(-E_{i} \beta\right)}{\sum_{j}^{N} \exp \left(-E_{j} \beta\right)}
$$

Here $N$ is number of sites in the lattice. The number of particles visiting to $i^{t h}$ site is defined by

$$
n_{i}=\frac{t_{i}}{\tau_{i}}=t_{n} \frac{\exp \left(E_{i, i+1} \beta\right)+\exp \left(-E_{i, i-1} \beta\right)}{2 \tau_{0} \sum_{j}^{N} \exp \left(-E_{j} \beta\right)}
$$

Therefore, the average time between two consecutive jumps in the limit of long time $t_{n}$ can be calculated as follow:

$$
\tau_{\text {jumpy }}=\frac{t_{n}}{n}=\frac{t_{n}}{\sum_{i}^{N} n_{i}}=\frac{2 \tau_{0} \sum_{i}^{N} \exp \left(-E_{i} \beta\right)}{\sum_{i}^{N} \exp \left(-E_{i, i+1} \beta\right)+\exp \left(-E_{i, i-1} \beta\right)}
$$

with the site and transition energies are adopted in accordance to Gaussian distribution (see Eq. (2)) 


\section{III.1. For the crystal case}

The site and transition energies are constant $\left(E_{i}=0 ; E_{i, i \pm 1}=0\right)$. Therefore, from the Eq. (10), it is deduced that:

$$
\begin{gathered}
\tau_{\text {jump } C}=\tau_{0} \\
\frac{\tau_{\text {jumpy }}}{\tau_{\text {jump } C}}=\frac{2 \sum_{i}^{N} \exp \left(-E_{i} \beta\right)}{\sum_{i}^{N} \exp \left(-E_{i, i+1} \beta\right)+\exp \left(-E_{i, i-1} \beta\right)}
\end{gathered}
$$

\section{III.2. For site disordered lattice}

The site energies $E_{i}$ are adopted in accordance to Gaussian distribution (see Eq. 2); and transition energies are constant $E_{i, i \pm 1}=0$. From Eq. (12) one obtains:

$$
\frac{\tau_{\text {jump } S D}}{\tau_{\text {jump } C}}=\sum_{i}^{N} \exp \left(-E_{i} \beta\right)
$$

Because the factor $F_{S}$ is independent of the distribution of site energies, $F_{S D}$ is always equal to 1.0. Therefore for SD, the diffusion coefficient can be determined by equations (6) and (13):

$$
\frac{D_{S D}}{D_{C}}=\frac{\tau_{\text {jumpC }}}{\tau_{\text {jump } S D}}=\frac{1}{\sum_{i}^{N} \exp \left(-E_{i} \beta\right)}
$$

\section{III.3. For transition disordered lattice}

The transition energies $E_{i, i \pm 1}$ are adopted in accordance to Gaussian distribution (see Eq.2); and site energies are constant $E_{i}=0$. From Eq. (12) one obtains:

$$
\frac{\tau_{\text {jump } T D}}{\tau_{\text {jump } C}}=\frac{2}{\sum_{i}^{N} \exp \left(-E_{i, i+1} \beta\right)+\exp \left(-E_{i, i-1} \beta\right)}
$$

As will be shown by simulation result in next section, the diffusion coefficient of TDL is close to the one of SDL if both lattices have the same temperature and energetic distribution. So, the diffusion coefficient of TDL is:

$$
\frac{D_{T D}}{D_{C}}=\frac{1}{\sum_{i}^{N} \exp \left(-E_{i, i+1} \beta\right)}
$$

From Eqs. (6) and (16) one obtains:

$$
F_{T D}=\frac{D_{T D}}{D_{C}} \frac{\tau_{\text {jumpTD }}}{\tau_{\text {jump }}}=\frac{2}{\sum_{i}^{N} \exp \left(-E_{i, i+1} \beta\right)\left[\sum_{i}^{N} \exp \left(-E_{i, i+1} \beta\right)+\sum_{i}^{N} \exp \left(-E_{i, i-1} \beta\right)\right]}
$$




\section{III.4. For mixed disordered lattice}

Both of the site and transition energies are randomly adopted in accordance to Gaussian distribution (see Eq.2). From Eq. (12) one obtains:

$$
\frac{\tau_{\text {jumpMD }}}{\tau_{\text {jump } C}}=\frac{2 \sum_{i}^{N} \exp \left(-E_{i} \beta\right)}{\sum_{i}^{N} \exp \left(-E_{i, i+1} \beta\right)+\exp \left(-E_{i, i-1} \beta\right)}
$$

Because the factor $F_{S}$ is independent of the distribution of site energies, $F_{S D}$ is always equal to 1.0. Therefore for MDL, $F_{M D}=F_{T D}$ can be calculated according to Eq. (17).

The diffusion coefficient can be determined via the Eqs. (6), (17) and (18):

$$
\frac{D_{M D}}{D_{C}}=F_{M D} \frac{\tau_{\text {jump }}}{\tau_{\text {jumpMD }}}
$$

in Fig. 1.

Distribution of site and transition energery in (a) SDL, (b) TDL and (c) MDL is presented

\section{RESULT AND DISCUSSION}

\section{IV.1. The single-particle case}

We have calculated the temperature dependence of the diffusion quantities of interest. The Monte-Carlo result is presented in figures 2, 3, 4 and 5. Here, the diffusion coefficient and correlation factor are determined by the slopes of the linear dependence of $\left\langle x_{n}^{2}\right\rangle$ versus $\left\langle t_{n}\right\rangle$ and $n$, respectively. In the case of the nonlinear dependence then nonlinear dependence will be divided into many linear sub-ranges and one can determined the slopes in the linear sub-ranges.

Fig. 2 shows the ratio $\tau_{j u m p S} / \tau_{j u m p C}$ as function of temperature for SDL and TDL. It can be seen that the time $\tau_{\text {jump } S} / \tau_{\text {jumpC }}$ is well approximated by Eqs. (13) and (15). For SDL, the time $\tau_{\text {jump } S} / \tau_{\text {jump } C}$ increases as temperature decreases (i.e. as temperature $\xi$ increases; $\xi \sim 1 / \mathrm{T}$ ) and the lower temperature decreases, the stronger $\tau_{\text {jump }} / \tau_{\text {jumpC }}$ increases. Conversely for TDL, the time $\tau_{\text {jumpS }} / \tau_{\text {jump } C}$ decreases as temperature decreases. Furthermore, the time $\tau_{\text {jump } S} / \tau_{\text {jump } C}$ of SDL is significantly larger than the one of TDL indicating the specific property of trapping model in comparison to hopping one.

Fig. 3 shows the temperature dependence of correlation factor $F$ that is calculated by both simulation and analytic methods (see Eq.(17)). For SDL, It can be seen that the correlation factor $F_{S}$ does not depend on temperature and is approximately equal to 1 . This is due to the factor $F$ is independent with the distribution of site energies. Conversely for TDL, the correlation factor significantly decreases as temperature decreases. The temperature dependence of diffusion coefficient for SDL and TDL systems is presented in figure 4. As expected, the ratio $D_{S} / D_{C}$ of

TDL is very close to the one of SDL if they have the same energetic distribution (see Eq. (2)) and temperature condition. The ratio $D_{S} / D_{C}$ decreases as temperature decreases. From figure 4 , we can see that it is in good agreement between simulation and analytical results for SDL and TDL. In order to check the validity of Eqs (17), (18) and (19), several simulations have been done for MDL. The result is presented in figure 5. One time again, it is in good agreement between simulation and calculation result. 

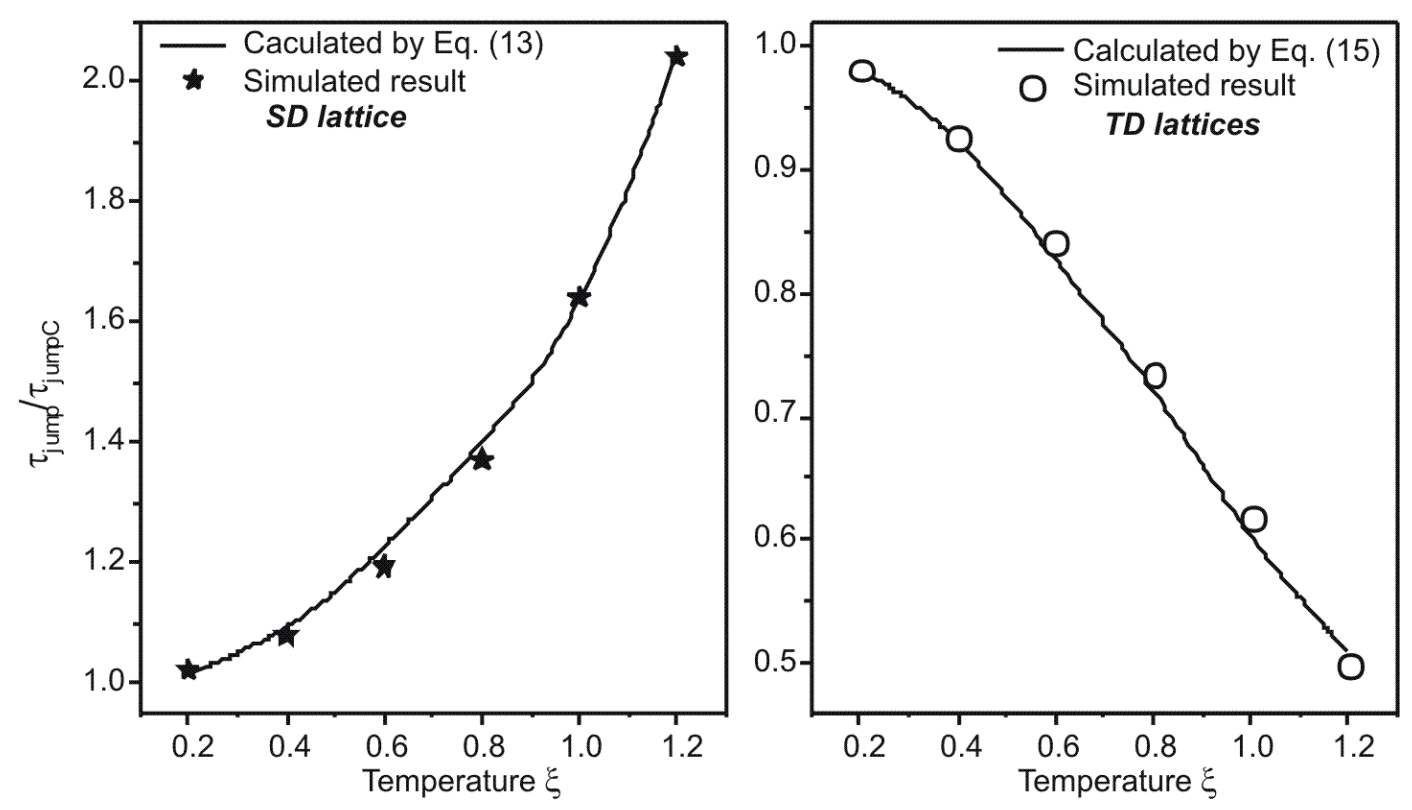

Fig. 2. The dependence of $\tau_{\text {jump }} / \tau_{j u m p C}$ on temperature for SDL, TDL model.

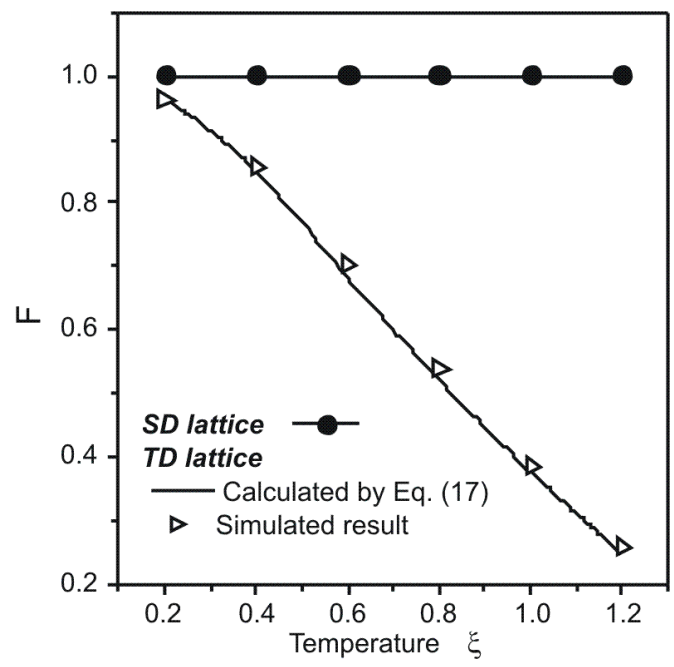

Fig. 3. The dependence of correlation factor $F$ on temperature for (solid circles) SD, (blank triangles) TD lattices.

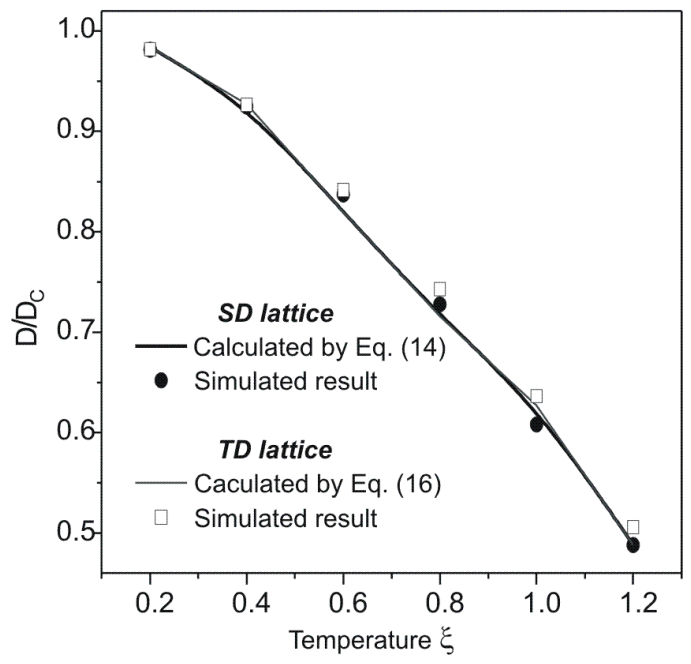

Fig. 4. The temperature dependence of $D / D_{C}$ for (solid circles) SDL and (blank squares) TDL.

\section{IV.2. Many-particle case}

For many-particle case, the blocking effect plays an important role in diffusion process. Unlike single-particle case, some particle's jumps in many-particle case are obstructed by other particles. In other word, the diffusion (displacement) of particle is blocked due to that a number of 
sites are occupied. Obviously, the number of such jumps (unsuccessful hop) $n_{\text {uns }}$ increases with the number of particles. Consequently, the mean square displacement and correlation factor

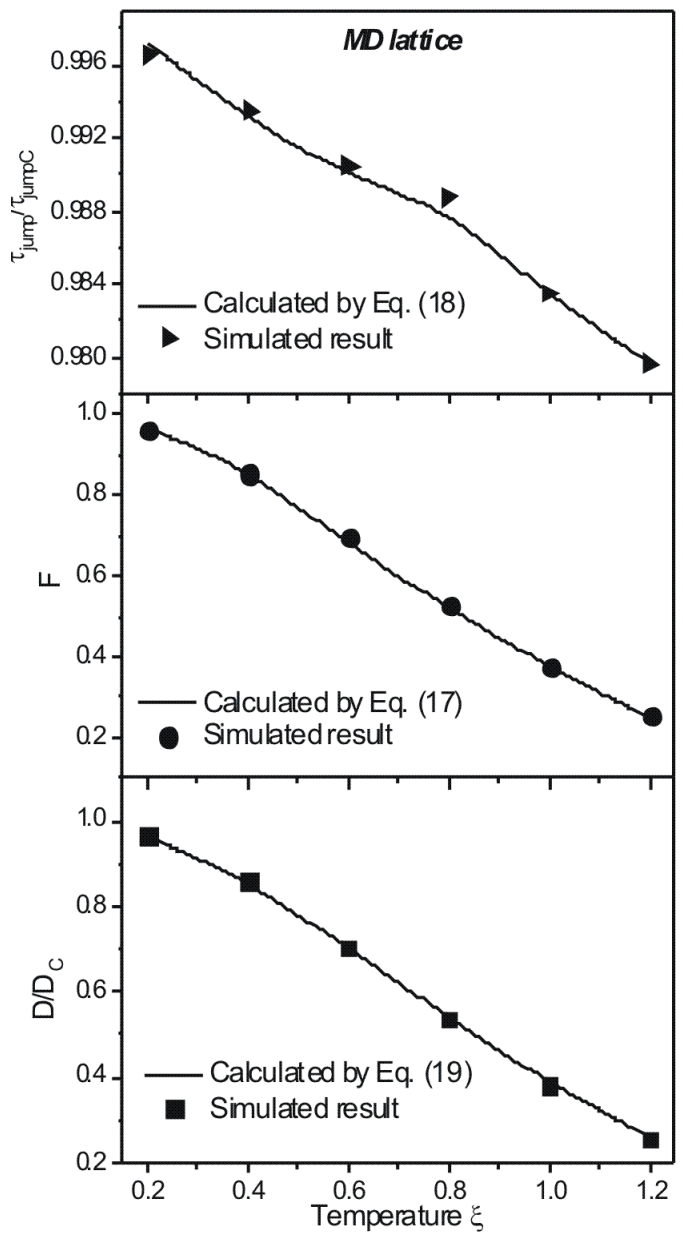

Fig. 5. The dependence of $\tau_{j u m p} / \tau_{j u m p C}$, correlation factor $\mathrm{F}$ and $\mathrm{D} / \mathrm{D}_{C}$ on temperature for MDL system.

$F_{M}$ decreased with increasing number of particles. Table 1 presents the diffusion quantities for many-particle case at temperature $\xi=1.2$. The number of unsuccessful hops $n_{\text {uns }}$ relates to the correlation factor $F_{M}$. As the number of particles increases, $n_{u n s} /$ nincreases about 14.25 times for SDL and 13.19 times for TDL systems, meanwhile the factor $\mathrm{F}_{M}$ decreases for both SDL (1.94 times) and TDL (1.47 times) systems. This effect is denoted to $F$-effect. This effect can be explained as follows: since the particle's hop is unsuccessful, the probability that the particle's hop in opposite direction becomes bigger than one in original direction. This gives rise to increasing the number of forward-backward hops and results in the decrease of correlation factor $F_{M}$ and final consequence is the decrease of diffusion coefficient $\mathrm{D}_{M}$. For SDL, the mean particle's residence time for the site with low energy is larger than the one for site with high energy. Therefore, the 


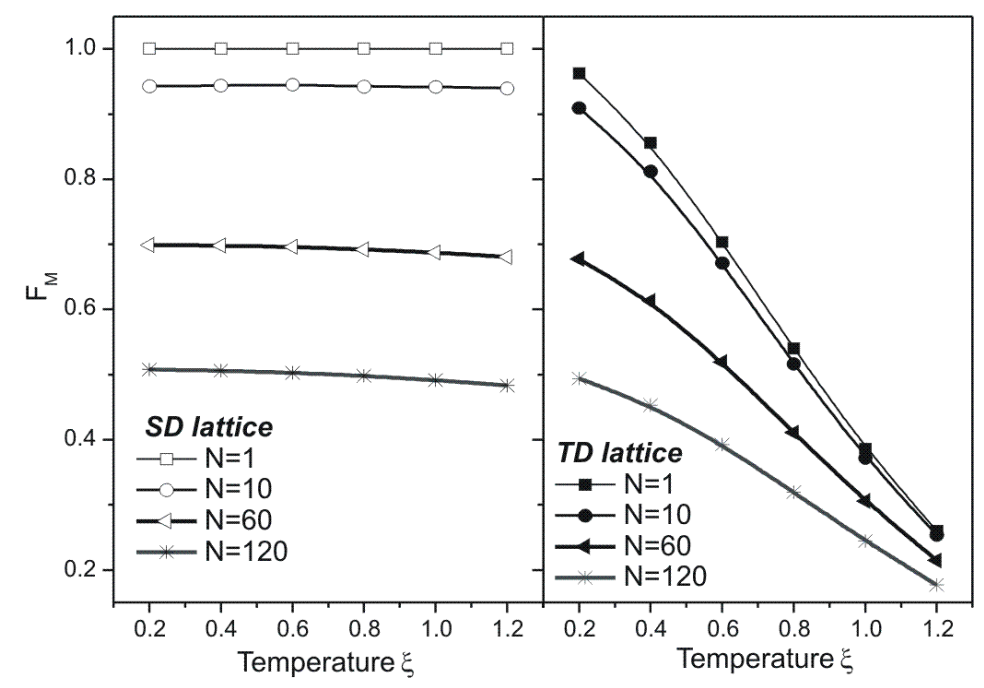

Fig. 6. The dependence of correlation factor $\mathrm{F}_{M}$ on temperature for SD (left) and TD (right) lattices

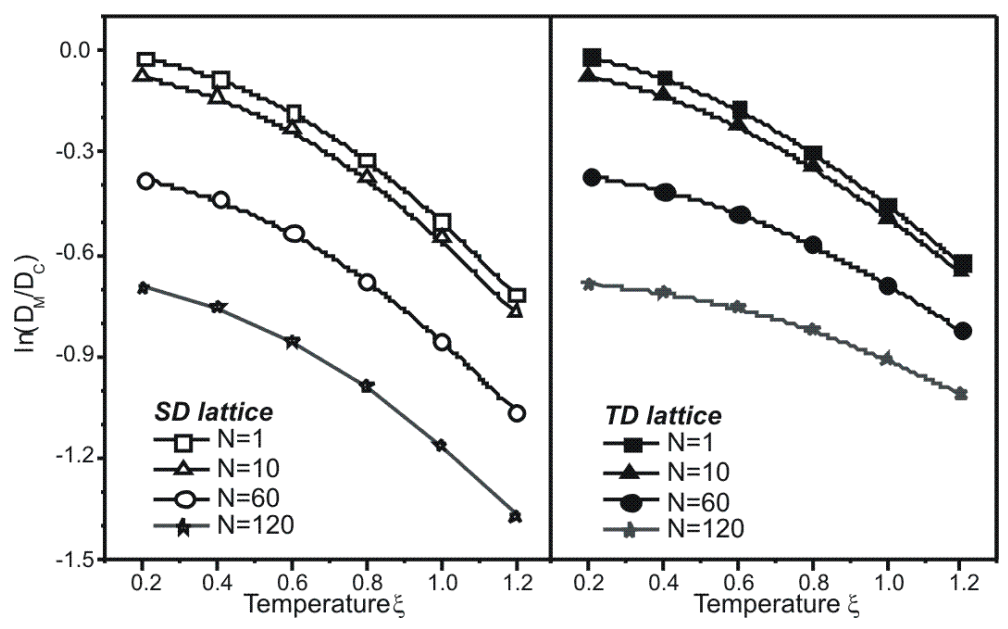

Fig. 7. The dependence of $\ln \left(\mathrm{D}_{M} / \mathrm{D}_{C}\right)$ on temperature for (left) SD and (right) TD lattices

occupied site with low energy prevents other particles from jumping into it for a time interval that is longer in comparison to the occupied site with higher energy. As a result, due to blocking, the average number of visits of particle to the site with low energy decreases with the density of particles. This leads to decreasing mean time between two consecutive hops $\tau_{\text {jump } M}$. This effect is called $\tau$-effect and gives rise to increasing $\mathrm{D}_{M}$. For TDL, the particle spent in average the same time for each site. However, it prefers to surmount the saddle points with low transition energy. Hence the particle's jumps over the saddle points with low transition energy are more frequent than the ones with high transition energy. As a result, due to blocking, the number of jumps over 
saddle point with low transition energy decreases when the number of particles is large enough. This results in increasing time $\tau_{\text {jumpM }}$.

Table 1. The diffusion quantities for many-particles at $\xi=1.2$ and $n=1000$. Here $n$ is average number of hops per particle; $n_{\text {high }}, n_{\text {low }}$ are the average number of visit to site with high and low energy, respectively; $n_{\text {high }},+n_{\text {low }}=n ; n_{\text {uns }}$ is number of unsuccessful jumps.

\begin{tabular}{cccccccc}
\hline \hline Lattice & $\boldsymbol{N}$ & $\boldsymbol{n}_{\text {high }}$ & $\boldsymbol{n}_{\text {low }}$ & $\boldsymbol{n}_{\text {uns }}$ & $\boldsymbol{F}_{M}$ & $\tau_{\text {jumpM }} / \tau_{\text {jumpC }}$ & $\boldsymbol{D}_{M} / \boldsymbol{D}_{C}$ \\
\hline \hline \multirow{6}{*}{ SD } & 1 & 699.43 & 301.57 & 0 & 1.001 & 2.043 & 0.488 \\
& 10 & 698.68 & 301.42 & 0.56 & 0.939 & 2.017 & 0.462 \\
\cline { 2 - 8 } & 20 & 699.67 & 300.38 & 1.18 & 0.879 & 2.003 & 0.435 \\
\cline { 2 - 8 } & 40 & 702.12 & 297.90 & 2.47 & 0.772 & 1.978 & 0.387 \\
\cline { 2 - 8 } & 60 & 704.31 & 295.71 & 3.80 & 0.681 & 1.954 & 0.346 \\
\cline { 2 - 8 } & 80 & 706.46 & 293.56 & 5.15 & 0.604 & 1.932 & 0.311 \\
\cline { 2 - 8 } & 120 & 710.83 & 289.18 & 7.98 & 0.483 & 1.889 & 0.254 \\
\hline \multirow{5}{*}{ TD } & 1 & - & - & 0 & 0.260 & 0.499 & 0.536 \\
\cline { 2 - 8 } & 10 & - & - & 2.26 & 0.254 & 0.490 & 0.520 \\
\cline { 2 - 8 } & 20 & - & - & 4.72 & 0.245 & 0.488 & 0.503 \\
\cline { 2 - 8 } & 40 & - & - & 9.75 & 0.229 & 0.488 & 0.470 \\
\cline { 2 - 8 } & 60 & - & - & 14.75 & 0.215 & 0.488 & 0.440 \\
\cline { 2 - 8 } & 80 & - & - & -29.82 & 0.201 & 0.489 & 0.412 \\
\hline \hline & 120 & - & - & 0.177 & 0.488 & 0.363 \\
\hline
\end{tabular}

Nevertheless, our simulation results show that $\tau_{\text {jumpM }}$ decreases (see Table 1). This can be explained as follows: in the considered range of the number of particle (from 10 to 120 particles) the blocking effect is weekly for TDL system due to the number of particles is not large enough. As expected, the average number of visits to site with high $n_{\text {high }}$ and the number of unsuccessful jumps $n_{\text {uns }}$ increases monotonously as the number of particles increases from 10 to 120 . This gives rise to decreasing $F_{M}$ and $\tau_{j u m p M}$. However, as shown table 1 the $D_{M} / D_{C}$ decreases with number of particle for all considered lattices. It implies that for one-dimensional lattices, the first effect $(F$-effect) is more dominant in comparison to the second one ( $\tau$-effect).

Fig. 6 shows that the temperature dependence of correlation factor $F_{M}$ for SDL and TDL systems. Similar to the case of single-particle, for SDL, the factor $F_{M}$ is independent on temperature but for TDL, the factor $\mathrm{F}_{M}$ is significantly dependent on temperature and decreases with temperature. To get more insight into the diffusive behaviors of many-particle system, we studied the temperature dependence of quantity $\ln \left(\mathrm{D}_{M} / \mathrm{D}_{C}\right)$ shown in figure 7 . From figure 7 , it can be seen that the diffusion does not obey Arrhenius relation for all cases. In accordance to ref. [15], the Arrhenius behavior for diffusion in amorphous material is caused by the compensation between site and transition disordered. This discrepancy maybe related to the finite energetic distribution used in [15]. To estimate the strength of blocking effect, we have calculated the $\operatorname{ratios} \mathrm{F}_{M} / \mathrm{F}_{S}$, $\tau_{\text {jump } M} / \tau_{\text {jump } S}$ and $\mathrm{D}_{M} / \mathrm{D}_{S}$ which are shown in figure 8. It can be seen that $\mathrm{F}_{M} / \mathrm{F}_{S}$ decreases with different rates and depends on the type of disorder and number of particles. Meanwhile for TDL, the value of $\tau_{\text {jumpM }} / \tau_{\text {jumpS }}$ is almost unchanged or slightly increases with the number of 
particles, for SDL, its value significantly decreases at temperature $\xi=1.2$; the ratio $D_{M} / D_{S}$ decreases with the number of particles for both SDL and TDL. As such, increasing the number of particles is accompanied with two effects: $F$-effect that leads to decreasing $D_{M}$ and $\tau$-effect that leads to increasing $D_{M}$. However, for TD lattice F-effect is mainly but for SD lattices $F$-effect is stronger than $\tau$-effect. Fig. 9 shows the temperature dependence of $F_{M} / F_{S}, \tau_{j u m p M} / \tau_{j u m p S}$ and $D_{M} / D_{S}$ for SDL and TDL. The temperature dependence of $F_{M} / F_{S}$ as well as $\tau_{\text {jump }} / \tau_{\text {jump}} S$ is quite different between SDL and TDL. In the considered temperature range, the ratio $F_{M} / F_{S}$ decreases from about 0.507 to 0.429 for SDL. Whereas, it increases from about 0.513 to 0.927 for TDL. Despite that the factor $F_{M}$ as well as the time $\tau_{j u m p M}$ significantly depends on the temperature, the ratio $D_{M} / D_{S}$ slightly changes in the considered temperature range. Therefore, the blocking effect weakly depends on the temperature.

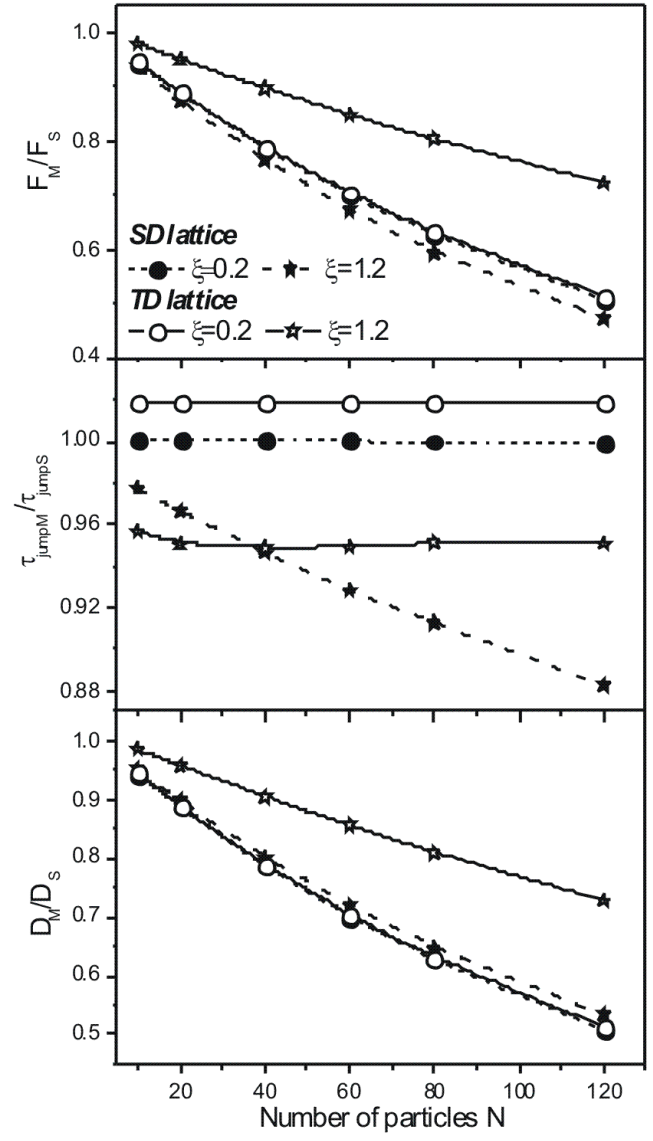

Fig. 8. The dependence of $\mathrm{F}_{M} / \mathrm{F}_{S}$, $\tau_{\text {jump } M} / \tau_{\text {jump } S}, \quad \mathrm{D}_{M} / \mathrm{D}_{S}$ on number of particles for (solid symbol) SD and (blank circles) TD lattices.

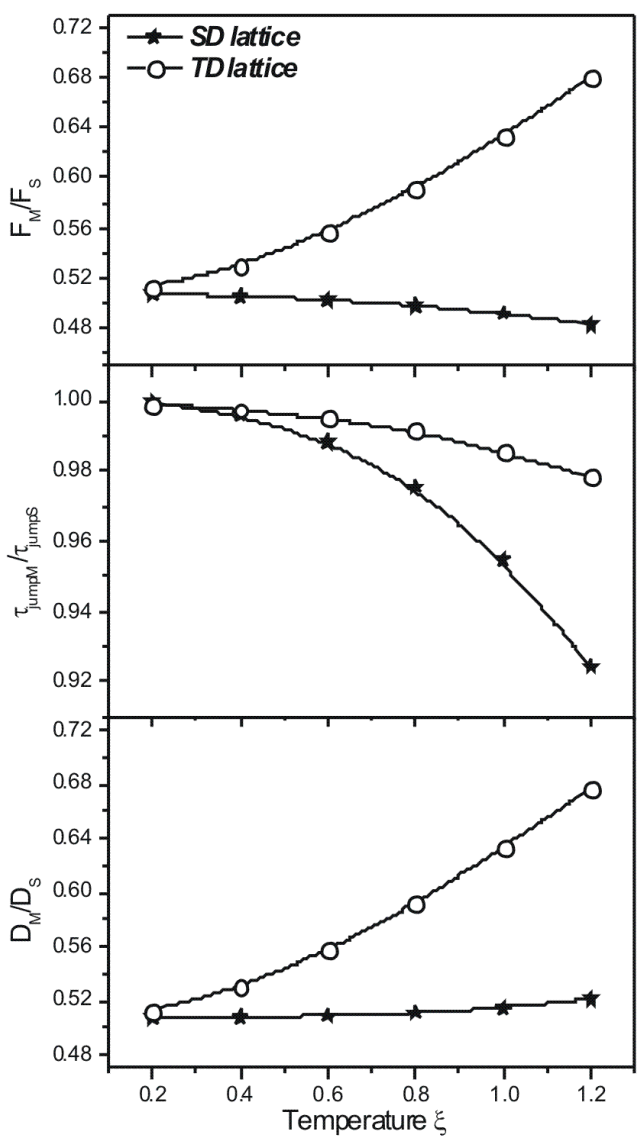

Fig. 9. The dependence of $\mathrm{F}_{M} / \mathrm{F}_{S}$, $\tau_{\text {jump } M} / \tau_{\text {jump } S}, \mathrm{D}_{M} / \mathrm{D}_{S}$ on temperature for (solid symbol) SDL and (blank circles) TDL systems. 


\section{CONCLUSIONS}

Monte-Carlo simulations were carried out to study the diffusion in one-dimensional disordered lattices with Gaussian distributions of site and transition energies. The main results show that: 1) in the case of single-particle, the diffusion coefficients of particles in SDL and TDL systems are identical if both systems have the same temperature and energetic distribution. However, the character of particle's motion in the systems is quite different. This result enables us to construct the analytic expressions for diffusion coefficient $D$, the time between two consecutive hops of particle $\tau_{\text {jump }}$ and correlation factor $F$ which show a good agreement between simulation and analytic calculation results. The diffusion coefficient $D$ is proportional to factor $F$ and $1 / \tau_{\text {jump }}$. For the SDL, with decreasing temperature, the diffusion coefficient $D$ decreases due to $\tau_{\text {jump }}$ increases. Conversely, factor $F$ is always equal to 1 and independent of temperature. For TDL and MDL, with decreasing temperature, the reduction of factor $F$ result in the decrease of diffusion coefficient $D$ and the decrease of $\tau_{\text {jump }}$ result in the increases of diffusion coefficient $D$. However, because the factor $F$ decreases more strongly than $\tau_{j u m p}$, this results in the decrease of diffusion coefficient $D$ as temperature decreases. 2) in the case of many particles, the results reveal two specific effects: $F$-effect and $\tau$-effect. With increasing the number of particles, the diffusion coefficient $D_{M}$ decreases for SDL and TDL due to $F$-effect is more dominant than $\tau$ effect. The Arrhenius behavior is not observed for all systems. It can be seen that blocking effect is significantly dependent on the number of particles but it slightly dependent on the temperature. In the considered range of the number of particles (from 10 to 120 particles) the blocking effect in SDL is more dominant than in TDL. The more number of particles the system has, the more clear the blocking effect reveals.

\section{ACKNOWLEDGEMENT}

This research is funded by Vietnam National Foundation for Science and Technology Development (NAFOSTED) under grant number 103.05-2013.30.

\section{REFERENCES}

[1] J.W. Haus, K.W. Kehr, Phys. Rep. 150 (1987) 263.

[2] Peter M.Richards, Phys.Rev.B,16 (1977) 4,1393.

[3] Li-Shi Luo et al., Phys.Rev.E, 51 (1995),1,43.

[4] A. V. Nenashev, F. Jansson, S. D. Baranovskii, R. Österbacka, A. V. Dvurechenskii, and F. Gebhard, Phys.Rev. $B, 81$ (2010), 115203.

[5] J.W.Van de Leur, A.Yu. Orlov, Phys. Lett. A, 373(31) (2009) 2675.

[6] Y. Limoge, J. L. Bocquet, J.non-cryst.solids, 117-118, Part 2, (1990), 605-608.

[7] Y.Limoge, J.L.Bocquet, Phys.Rev.Lett.65 (1990), 1, 60.

[8] Panos Argyrakis et al, Phys.Rev. E, 52 (1995) ,4, 3623.

[9] A. Tarasenko, L. Jastrabik, Applied Surface Science 256 (2010), 5137

[10] S. H. Payne and H. J. Kreuzer,Phys. Rev. B 75 (2007), 115403.

[11] T. Apih, M. Bobnar, J. Dolinsěk, L. Jastrow, D. Zander, U. Köster, Solid State Commun. 134 (2005), 337.

[12] N. Eliaz, D. Fuks, D. Eliezer, Mater. Lett. 39 (1999), 255.

[13] V. V. Kondratyev, A.V.Gapontsev, A.N. Voloshinskii, A.G. Obukhov, N.I.Timofeyev, Inter. J. of Hydrogen Energy 24 (1999) 708.

[14] Y. Limoge, J. L. Bocquet, Acta metal. 36 (1988), 7, 1717.

[15] R. Kutner, Physica A 224 (1996) 558. 